\title{
Politicas públicas para a população idosa: uma revisão com ênfase nas ações de saúde
}

\section{Public policies for the elderly population: a review with emphasis on healthcare actions}

\author{
Marina Picazzio Perez Batista ${ }^{1}$, Maria Helena Morgani de Almeida ${ }^{2}$, \\ Selma Lancman ${ }^{3}$
}

\begin{abstract}
BATISTA, M. P. P.; ALMEIDA, M. H. M.; LANCMAN, S. Politicas públicas para a população idosa: uma revisão com ênfase nas ações de saúde. Rev. Ter. Ocup. Univ. São Paulo, v. 22, n. 3, p. 200-207, set./dez. 2011.
\end{abstract}

RESUMO: O envelhecimento populacional é um fenômeno mundial que impõe grandes desafios, especialmente aos países em desenvolvimento e demanda a implementação de Políticas Públicas específicas. Este artigo, que é parte integrante do projeto de dissertação de mestrado intitulada "Reflexões sobre o cotidiano de trabalho do profissional acompanhante de idoso do Programa Acompanhante de Idosos- PAI", apresenta os principais documentos internacionais e nacionais que objetivaram nortear a implementação de programas e Políticas destinados aos idosos com ênfase nas ações de responsabilidade do setor Saúde. Tais documentos reconhecem os idosos como sujeitos de direito e valorizam o estímulo à independência, autonomia, participação social e convívio familiar e comunitário.

DESCRITORES: Politicas públicas de saúde; Políticas; Envelhecimento; Idoso.

1. Terapeuta Ocupacional formada pela Universidade de São Paulo- Faculdade de Medicina. Departamento de Fonoaudiologia, Fisioterapia e Terapia Ocupacional.

2. Profa. Dra. do Departamento de Fonoaudiologia, Fisioterapia e Terapia Ocupacional da Faculdade de Medicina da Universidade de São Paulo.

3. Profa. Titular do Departamento de Fonoaudiologia, Fisioterapia e Terapia Ocupacional da Faculdade de Medicina da Universidade de São Paulo.

Endereço para correspondência: Rua Cipotânea, 51. Cidade Universitária 05360-000 - São Paulo - SP- Brasil. marinapperez@yahoo. com.br 


\section{INTRODUÇÃO}

$\mathrm{O}$ aumento da proporção de pessoas com 60 anos ou mais é um fenômeno mundial que se deve principalmente a uma redução nas taxas de fertilidade e aumento da expectativa de vida. Estima-se que até 2050, existirá no mundo aproximadamente dois bilhões de pessoas nesta faixa etária, sendo $80 \%$ nos países em desenvolvimento. Até 2025 o Brasil será o sexto país do mundo em número de idosos (OMS, 2005).

Algumas importantes particularidades do processo de envelhecimento populacional são: o crescente aumento do número de idosos com mais de 80 anos e o fenômeno da feminilização da velhice, ou seja, maior quantidade de mulheres do que homens neste grupo (OMS, 2005).

Nos países em desenvolvimento esse fenômeno têm imposto grandes desafios pois ocorreu de modo acelerado ao contrário dos países desenvolvidos, nos quais o envelhecimento da população foi um processo gradual (OMS, 2005). Além disso, esse crescimento acelerado tem sido acompanhado por mudanças nos padrões de trabalho e aumento da urbanização e da migração de jovens para cidades à procura de oportunidades de emprego e maior participação de mulheres no mercado de trabalho. Como resultado, uma proporção cada vez maior de pessoas está envelhecendo nas zonas urbanas, com pouco ou nenhum acesso à moradia e serviços. Ao mesmo tempo, existe grande número de pessoas em processo de envelhecimento residindo nas zonas rurais, em condições de solidão e distantes de seu ambiente familiar tradicional (ONU, 2003; OMS, 2005).

Outro desafio enfrentado pelos países em desenvolvimento é a carga dupla de doenças, que engloba tanto as doenças infecciosas e transmissíveis quanto as doenças crônicas não transmissíveis que, com o envelhecimento, transformam-se nas principais causas de incapacidade, morbidade e mortalidade (OMS, 2005).

O Brasil (1999) tem experimentado uma transição epidemiológica, com alterações relevantes no quadro de morbimortalidade. Em menos de 40 anos, o Brasil passou de um perfil de morbimortalidade típico de uma população jovem, para um caracterizado por enfermidades crônicas, próprias das faixas etárias mais avançadas, com custos diretos e indiretos mais elevados.

No Brasil as pessoas com 60 anos ou mais representam $11 \%$ da população total, com predomínio de mulheres (IBGE, 2010). Segundo dados da Pesquisa Nacional Por Amostra de Domicílios (IBGE, 2009), a maior concentração de idosos encontra-se no sudeste do país com $12,7 \%$ do total da população desta região. Em termos de Unidades da
Federação, os Estados do Rio de Janeiro, Rio Grande do Sul e São Paulo são os que apresentam maior porcentagem de idosos, apresentando respectivamente, $14,9 \%, 13,5 \%, 11,9 \%$ de suas populações.

No que tange à cidade de São Paulo, identifica-se, em relação ao gênero, a predominância de mulheres $(58,6 \%)$ e em relação à escolaridade, $21 \%$ das pessoas com 60 anos ou mais nunca freqüentaram a escola e $46,4 \%$ tem menos de 4 anos de estudo (SABE, 2003).

Em relação à rede de suporte, a maioria dos idosos é casado $(57 \%)$ e há uma parcela significativa de viúvos (29,5\%), com predominância de homens entre os idosos casados e mulheres entre as viúvas, dado que pode ser explicado pela maior expectativa de vida das mulheres, bem como pelo fato dos homens se casarem mais tardiamente e terem maior facilidade de recasamentos. A maioria vive acompanhada, porém, $30 \%$ com uma única pessoa, e desses, mais da metade reside com filhos, na maioria não casados, e aproximadamente um quarto com cônjuges. Os que moram sozinhos representam $13 \%$ observando-se neste grupo um aumento da proporção de idosos com idade avançada. Identificou-se também que são os membros do próprio domicílio que, com maior freqüência, prestam ou recebem ajuda dos idosos, seguido dos filhos que moram fora do domicílio (SABE, 2003).

Em relação à perda das habilidades funcionais, foi identificado que estas ocorrem em primeiro lugar nas atividades instrumentais e posteriormente nas básicas de vida diária, com pior desempenho nas instrumentais em idades mais avançadas, o que resulta em uma tendência ao isolamento domiciliar. O comprometimento dos idosos no desempenho das atividades de vida diária também foi associado ao aumento da idade, bem como ao gênero, com predomínio de declínio no sexo feminino. Em relação às dificuldades para as atividades básicas de vida diária, a maioria dos idosos $(80,7 \%)$ não apresentou dificuldades, porém uma parcela significativa $(30 \%)$ apresentou comprometimento em 7 ou mais atividade de vida diária, que implica em sobrecarga familiar e para o sistema de saúde. Foi observado um desequilíbrio entre a demanda dos idosos por auxílio para a realização das atividades básicas de vida diária e aquele efetivamente recebido, em especial para as mulheres (SABE, 2003).

Assim como o observado em São Paulo, há o reconhecimento mundial de que a incapacidade para realização de atividades cotidianas devido às doenças crônicas aumenta conforme a idade. As mulheres são o grupo de maior risco para incapacidades devido: à diferença entre os gêneros no que se refere à propensão à doenças, à maior expectativa de vida das mulheres, à maior proporção de mulheres viúvas e vítimas de violência doméstica e às 
desigualdades por razão de gênero sofridas ao longo da vida (ONU, 2003; OMS, 2005).

As incapacidades podem conduzir o indivíduo à uma condição de dependência, à equipamentos específicos ou ajuda de outrem para a realização de atividades cotidianas. Neste contexto é imprescindível a figura do cuidador, caracterizado como o indivíduo, membro ou não da família, que, com ou sem remuneração, cuida do idoso doente ou dependente no exercício de suas atividades diárias, excluindo-se da realização de procedimentos e técnicas de atribuição de profissões legalmente estabelecidas, especialmente da área da enfermagem (BRASIL, 1999).

Neste sentido é possível caracterizar o apoio prestado aos idosos em cuidado formal ou informal. Os sistemas formais de cuidado são constituídos por profissionais e instituições que realizam assistência sob a forma de prestação de serviço, ou seja, pessoa ou agências comunitárias contratadas para tal finalidade. Os sistemas informais compreendem pessoas da família, amigos e vizinhos, que fornecem apoio e cuidados voluntários ao idoso com incapacidade funcional (BRASIL, 1999).

Mundialmente, os sistemas informais são essenciais para o cuidado com os idosos dependentes. Entretanto os cuidadores informais, frequentemente mulheres idosas, devem ser amparados na prestação de auxílio aos idosos e no cuidado consigo mesmo, uma vez que é reconhecido que a tarefa de cuidar pode levar ao adoecimento do próprio cuidador. Para tanto, é essencial o fornecimento de informações que amparem os cuidadores no cuidado, bem como a garantia de acesso à serviços de apoio (OMS, 2005).

No que tange à realidade brasileira, é preciso levar em consideração, na formulação das Políticas para este segmento populacional, mudanças sociais que atualmente influenciam diretamente o suporte informal aos idosos, em especial às modificações estruturais da família. A família, tradicionalmente considerada o mais eficaz sistema de apoio aos idosos, têm modificado bastante sua estrutura em decorrência da: contínua migração dos mais jovens, do número crescente de divórcios e segundo ou terceiro casamento e do aumento no número de famílias em que a mulher exerce o papel de chefe (BRASIL, 1999).

Em articulação com o cuidado informal, o sistema formal deve ser fortalecido e acessível para todos, destacando-se seu engajamento às diversas modalidades de atenção: cuidado domiciliar, serviços comunitários, serviços de reabilitação e hospitais-dia. A fim de estimular a independência e autonomia dos idosos, é importante também a garantia da provisão de equipamentos de auxílio e a capacitação de cuidadores e profissionais em conteúdos referentes à Gerontologia (OMS, 2005).
Considerando os desafios impostos pelo processo de envelhecimento populacional e pela relevância do tema, este artigo, que é parte integrante do projeto de dissertação de mestrado intitulada "Reflexões sobre o cotidiano de trabalho do profissional acompanhante de idoso do Programa Acompanhante de Idosos- PAI", apresenta os principais documentos internacionais e nacionais que objetivaram nortear a implementação de programas e Políticas destinados aos idosos com ênfase nas ações de responsabilidade do setor Saúde.

\section{DOCUMENTOS INTERNACIONAIS SOBRE O ENVELHECIMENTO}

A fim de fornecer instrumentos práticos para auxiliar na formulação de Políticas para a população idosa, dois importantes documentos elaborados pelas organizações internacionais são destacados por apresentarem prioridades básicas associadas ao envelhecimento: o "Plano de Ação Internacional para o Envelhecimento" (ONU, 2003) e o documento "Envelhecimento Ativo: Uma Política de Saúde" (OMS, 2005), os quais serão melhor detalhados a seguir.

\subsection{Plano de Ação Internacional para o Envelhecimento}

O Plano de Ação Internacional para o Envelhecimento (ONU, 2003) foi resultado da II Assembléia Mundial do Envelhecimento, realizada em 2002 em Madrid, e tem como finalidade garantir o envelhecimento dos indivíduos com segurança, dignidade e oportunidades de participação social e promover o desenvolvimento de uma sociedade para todas as idades. Para tanto, recomenda que em todos os países se adotem medidas em três direções prioritárias: promoção da saúde e bem-estar na velhice, participação ativa dos idosos no desenvolvimento de sua sociedade e criação de um ambiente propício e favorável que realce as capacidades, habilidades e contribuições dos idosos.

Para viabilização das medidas propostas foram sugeridas, ao longo do documento, a adoção de estratégias dentre as quais destacam-se: a) Incorporação da perspectiva de gênero em todas as Políticas e programas, reafirmando a igualdade dos sexos; b) Promoção e proteção dos direitos humanos e das liberdades fundamentais; c) Criação de medidas para erradicação da pobreza; d) Prestação de auxílio aos idosos pertencentes a populações indígenas, migrantes, residente em zonas rurais, em situações de conflito e ocupação estrangeira, idosos com AIDS e aqueles com questões de saúde mental; e) Garantia de acesso universal à assistência e serviços de saúde; f) Fortalecimento da solidariedade entre as gerações; g) Capacitação de idosos, a fim de viabilizar sua participação ativa nos âmbitos 
econômicos, políticos e sociais, incluindo oportunidades de trabalho remunerado e voluntário; h) Aproveitamento do potencial e dos conhecimentos de idosos nos aspectos social, cultural e educativo; i) Garantia de oportunidades de desenvolvimento, realização pessoal e bem-estar, bem como de direitos econômicos, sociais, culturais, civis e políticos; j) Eliminação de todas as formas de violência e discriminação contra idosos; k) Redução dos efeitos acumulativos dos fatores que aumentam o risco de doenças e dependência dos idosos, visando a manutenção da máxima capacidade funcional; 1) Capacitação de prestadores de serviços de saúde e de profissionais de Saúde quanto às necessidades de idosos; m) Garantia de condições para que os idosos vivam o maior tempo possível de forma independente em sua comunidade; n) Estabelecimento de condições que favorecem às famílias e comunidade a proverem cuidados e proteção aos idosos, a partir da promoção de medidas de assistência comunitária e apoio à atenção familiar, organizando sistemas de apoio formais que incluam a assistência a longo prazo ao idosos frágeis em seu domicílio (ONU, 2003).

A importância da adoção de Políticas para a viabilização do Plano Internacional de Ação sobre o Envelhecimento (ONU, 2003) foi reafirmada na $26^{\mathrm{a}}$ Conferência Sanitária Pan-americana, em sua sessão Saúde e o Envelhecimento (OMS, 2002). Esta Conferência reiterou, ainda, a necessidade de promoção e proteção dos direitos humanos dos idosos, promoção e ampliação de seu acesso à atenção de saúde e o desenvolvimento de alternativas de atenção comunitária a longo prazo (OMS, 2002).

\subsection{Envelhecimento ativo: saúde, participação e segurança}

A Política do Envelhecimento Ativo (OMS, 2005) objetiva primordialmente aumentar a qualidade de vida dos indivíduos que envelhecem, incluindo aqueles que possuem incapacidades e requerem auxílio para realização de suas atividades cotidianas. Por qualidade de vida entende-se:

A percepção que o indivíduo tem de sua posição na vida dentro do contexto de sua cultura e do sistema de valores de onde vive, e em relação a seus objetivos, expectativas, padrões e preocupações. É um conceito muito amplo que incorpora de uma maneira complexa a saúde física de uma pessoa, seu estado psicológico, seu nível de dependência, suas relações sociais, suas crenças e sua relação com características proeminentes no ambiente (OMS, 1994).

O Envelhecimento Ativo caracteriza-se pelo processo de otimização das oportunidades de saúde, segurança e participação, direcionados para indivíduos ou grupos populacionais. A palavra ativo se remete à participação contínua nas questões econômicas, culturais, civis, sociais e espirituais, reconhecendo-se os idosos como sujeito de direitos (OMS, 2005).

A Política do Envelhecimento Ativo (OMS, 2005) tem a saúde como um de seus pilares básicos, e as ações neste âmbito devem considerar a manutenção em níveis baixos dos fatores de risco ambientais e comportamentais para doenças crônicas e declínio funcional e elevação dos fatores de proteção. Outro aspecto relevante é o apoio aos cuidadores informais por meio do fornecimento de orientações para facilitação do cuidado e de pensões e subsídios financeiros, bem como estimulando-se a criação de serviços em domicílio e hospital-dia. A Política também ressalta a importância do apoio aos cuidadores formais, particularmente àqueles não especializados, com baixa posição social e profissional, com a promoção de condições adequadas de trabalho e de remuneração, e oferta de treinamento e educação na área do envelhecimento (OMS, 2005).

No âmbito da participação, o Envelhecimento Ativo propõe o incentivo à educação e oportunidades de aprendizagem durante o curso da vida, envolvimento ativo dos idosos nas atividades de trabalho formal, informal e voluntário e sua participação, de forma integral, na vida familiar e comunitária. No que tange à segurança, aponta para a garantia de proteção, segurança e dignidade aos idosos, por meio de reconhecimento de direitos e necessidades de segurança física, financeira, social (OMS, 2005).

O Envelhecimento Ativo tem como princípios relevantes: o incentivo à interdependência e solidariedade entre gerações; a criação de ambientes amistosos para os idosos; a redução de iniqüidades entre homens e mulheres; a extinção de formas de discriminação de idade; o reconhecimento da diversidade das populações em processo de envelhecimento; a promoção de ações intersetoriais; a manutenção da autonomia e independência dos idosos (OMS, 2005).

O conceito de autonomia é definido como a habilidade de lidar e tomar decisões pessoais sobre como se deve viver, conforme suas próprias regras e preferências. Já o conceito de independência refere-se à habilidade de executar, sem auxílio, as funções relacionadas à vida diária (OMS, 2005).

O Envelhecimento Ativo reconhece os idosos em sua diversidade e a heterogeneidade dos fatores envolvidos no envelhecimento. A cultura e o gênero são os fatores transversais que influenciam diretamente o modo como os indivíduos envelhecem e se articulam com os demais fatores: relacionados aos sistemas de saúde e serviço social; comportamentais, relacionados à participação ativa no cuidado da própria saúde e adoção de estilos de vida saudáveis; pessoais, advindos da biologia e genética 
e aspectos psicológicos; relacionados ao ambiente, como moradia segura e acesso a água limpa, ar puro e alimentos seguros; relacionados ao ambiente social, tais como o apoio social, oportunidades de educação e aprendizagem permanente, paz, e proteção contra a violência e maus-tratos; econômicos, particularmente os que se remetem à renda, ao trabalho, e à proteção social (OMS, 2005).

Especificamente no que se refere aos fatores relacionados aos sistemas de saúde o documento afirma a necessidade da promoção da saúde, prevenção de doenças e acesso eqüitativo aos cuidados primário e de longo prazo, entendendo como assistência a longo prazo aquela prestada por cuidadores informais e/ou formais à indivíduos com incapacidades para seu autocuidado, englobando serviços comunitários e instituições de longa permanência (OMS, 2005).

\section{POLÍTICAS PÚBLICAS BRASILEIRAS PARA A POPULAÇÃO IDOSA}

A partir da Constituição Federal de 1988 (BRASIL, 1988), os idosos começaram a ser alvo das Políticas Públicas. A Constituição tem como fundamentos a cidadania e a dignidade da pessoa humana, e objetiva promover o bem de todos e eliminar quaisquer formas de discriminação ou de distinção de qualquer natureza, o que inclui as de sexo e idade. Em seu artigo $5^{\circ}$ afirma que todos são iguais perante a lei e garante o direito à vida, à liberdade, à igualdade, à segurança e à propriedade (BRASIL, 1988).

Em relação ao amparo específico aos idosos, a Constituição atribui tal dever à família, à sociedade e ao Estado, de modo a assegurar sua participação na comunidade, dignidade, bem-estar e direito à vida. No âmbito familiar, cabe aos filhos maiores amparar os pais na velhice, carência ou enfermidade.

Visando a proteção desta população, o texto constitucional prevê que os programas de amparo aos idosos deverão ser executados preferencialmente em seus lares. Aos idosos que não possuírem meios de prover à própria manutenção ou de tê-la garantida por sua família, fica assegurado o direito de recebimento de benefício mensal de um salário mínimo (BRASIL, 1988).

De acordo com a Constituição (BRASIL, 1988), a Política Nacional do Idoso (BRASIL, 1994, 1996) reitera o dever da família, sociedade e Estado em assegurar os direitos sociais do idoso, criando condições para integração e participação efetiva na sociedade e promoção de sua autonomia, vetando qualquer forma de discriminação contra a pessoa idosa.

Esta Política foi criada a partir da lei $\mathrm{n}^{\circ} 8.842$ e regulamentada pelo decreto $\mathrm{n}^{\circ} 1.948$ (BRASIL, 1994,
1996). Entre suas diretrizes estão a viabilização de modos alternativos de participação, ocupação e convívio do idoso e o estímulo à intergeracionalidade. Para tanto incentiva a implementação de centros de convivência, centros-dia, hospitais-dia, casas-lares, oficinas abrigadas de trabalho e atendimento domiciliar (BRASIL, 1994, 1996). O atendimento domiciliar, realizado por profissionais da área de saúde ou por pessoas da própria comunidade, é a modalidade assistencial destinada ao idoso em condições de dependência e que vive só, prestada em seu próprio lar, com a finalidade de suprir suas necessidades da vida diária (BRASIL, 1996).

Outra diretriz adotada pela Política é a priorização do atendimento ao idoso no seio de suas próprias famílias, em detrimento do atendimento asilar, exceto àqueles que não apresentem condições que garantam sua própria sobrevivência, como nos casos de abandono, inexistência do grupo familiar ou casa-lar ou carência de recursos financeiros próprios ou da própria família (BRASIL, 1994, 1996, 2003). O conceito de modalidade asilar compreende o atendimento, em regime de internato ao idoso que não possua vínculo familiar ou condições de prover à própria subsistência (BRASIL, 1996).

No decreto $\mathrm{n}^{\circ} 1.948$ são detalhadas as atribuições do Ministério da Saúde, dentre as quais destacam-se: a) garantir ao idoso à assistência integral à saúde nos diversos níveis de atendimento do Sistema Único de Saúde, hierarquizada a partir das Unidades Básicas e da implantação da Unidade de Referência; b) estimular a participação do idoso nas diversas instâncias de controle social do Sistema Único de Saúde; c) favorecer o autocuidado e o cuidado informal do idoso; d) incentivar a permanência do idoso em sua comunidade (BRASIL, 1996).

A mesma lei que dispôs sobre a Política Nacional do Idoso (BRASIL, 1994), também criou o Conselho Nacional do Idoso, definindo que os Conselhos Nacional, Estaduais, do Distrito Federal e Municipais do Idoso constituir-se-ão em órgãos permanentes, paritários e deliberativos, compostos por igual número de organizações representativas da sociedade civil ligadas à área e representantes dos órgãos e entidades públicas.

Posteriormente, através da Portaria 1395/GM, foi criada a Política de Saúde do Idoso (BRASIL, 1999). São propósitos desta Política: a promoção do envelhecimento saudável e a melhoria e/ou manutenção ao máximo da capacidade funcional, a fim de garantir aos idosos permanência no meio em que vivem de forma independente. Por envelhecimento saudável se compreende o processo de envelhecimento com preservação da capacidade funcional, qualidade de vida e autonomia, a partir de ações que orientem os indivíduos para a adoção precoce de hábitos saudáveis 
de vida e a eliminação de comportamentos nocivos à saúde. Já a capacidade funcional é entendida como a capacidade de o indivíduo manter suas habilidades físicas e mentais requeridas para uma vida independente e autônoma (BRASIL, 1999).

Para viabilização da assistência às necessidades de saúde do idoso, a Política (BRASIL, 1999) pressupõe ações em diferentes âmbitos: ambulatorial, a partir da consulta geriátrica como a base dessa assistência; hospitalar com o estabelecimento de critérios específicos de atendimento por meio do estado funcional; internação hospitalar de longa permanência destinada a idosos com graves problemas de saúde, sem possibilidade de recuperação ou sujeito à recuperação prolongada; hospital-dia geriátrico para idosos cuja necessidade terapêutica - reabilitação, uso de medicação endovenosa, hidratação e quimioterapia - não justifiquem a permanência em hospital; outros serviços alternativos à internação prolongada, nos quais a assistência domiciliar deverá estar obrigatoriamente incluída (BRASIL, 1999).

$\mathrm{Na}$ modalidade de assistência domiciliar estão compreendidas a visitação domiciliar e cuidados domiciliares aos idosos com capacidade funcional comprometida, os quais englobam desde o fornecimento de equipamentos, até ações terapêuticas mais complexas. Relevante ressaltar que o fortalecimento do modelo de cuidados domiciliares não deverá objetivar a redução de custos ou transferências de responsabilidades, mas de modo contrário, deve-se intentar para o fornecimento de orientação, informação e assessoria de especialistas visando otimizar o suporte informal. Nesse sentido, nos cuidados domiciliares devese buscar desenvolver uma parceria entre os profissionais da saúde e as pessoas responsáveis pelos cuidados diretos ao idoso, com vistas à qualificação da assistência. Ainda, nessa modalidade propõe-se prestar cuidados ao próprio cuidador, reconhecendo-se que a tarefa de cuidar de um idoso dependente é desgastante e implica riscos à saúde do cuidador (BRASIL, 1999).

A Política também prevê para sua viabilização, a capacitação de recursos humanos especializados em Gerontologia e a articulação intersetorial do Ministério da Saúde com os Ministério da: Educação, Previdência e Assistência Social, Trabalho e Emprego, Justiça, Esporte e Turismo, Ciência e Tecnologia e Secretaria de Estado do Desenvolvimento Urbano (BRASIL, 1999).

Em 2003, por meio da lei $\mathrm{n}^{\circ} 10.741$, foi criado o Estatuto do Idoso que tem como objetivo assegurar facilidades e oportunidades para preservação da saúde física e mental, aperfeiçoamento moral, espiritual, intelectual e social dos idosos. Para tanto, atribui à família, comunidade, sociedade e Poder Público a obrigação de garantir ao idoso a efetivação de seus direitos, a saber: os direitos à vida, cidadania, liberdade, dignidade, alimentação, saúde, trabalho, cultura, educação, lazer, esporte e convivência familiar e comunitária. Reafirma ainda, o texto constitucional (BRASIL, 1988) delineando que nenhum idoso poderá sofrer qualquer tipo de discriminação, negligência, opressão, violência ou crueldade.

É prioridade do Estatuto (BRASIL, 2003), tal como colocado na Política Nacional do Idoso (BRASIL, 1999), a viabilização de formas alternativas de participação e convívio do idoso com outras gerações e a capacitação dos recursos humanos nas áreas de geriatria e gerontologia. Outra prioridade é o atendimento do idoso por sua própria família, em detrimento do atendimento asilar.

$\mathrm{Na}$ que tange, especificamente ao direito à saúde, o Estatuto prevê ações e serviços no sentido de favorecer a prevenção, promoção, proteção e recuperação da saúde visando a atenção integral à saúde do idoso. Cabe às instituições de saúde promover o treinamento e a capacitação em Gerontologia aos seus profissionais, bem como fornecer orientação a cuidadores familiares e promover grupos de auto-ajuda. $\mathrm{O}$ Estatuto reitera a importância da atenção à saúde, na forma de atendimento domiciliar, incluindo a internação, a ser prestado aos idosos que dele necessitarem e que estejam impossibilitados de se locomover ou para os idosos abrigados em instituições (BRASIL, 2003).

Posteriormente, no ano de 2006, por meio da Portaria $\mathrm{n}^{\circ} 2.528$, foi aprovada a Política Nacional de Saúde da Pessoa Idosa cuja finalidade é manter, recuperar e promover a autonomia e a independência dos indivíduos idosos. Como diretrizes aponta para: a promoção do Envelhecimento Ativo e saudável, seguindo as orientações do documento "Envelhecimento Ativo: Uma Política de Saúde" (OMS, 2005), estímulo à participação e fortalecimento do controle social e atenção integral e integrada à saúde da pessoa idosa (BRASIL, 2006).

Visando à integralidade da atenção, a Política Nacional de Saúde da Pessoa Idosa propõe a articulação entre os setores: Educação, Previdência Social, Sistema Único de Assistência Social, Trabalho e Emprego, Desenvolvimento Urbano, Transportes, Justiça e Direitos Humanos, Esporte e Lazer, Ciência e Tecnologia (BRASIL, 2006).

Segundo a mesma Política (BRASIL, 2006), dois eixos norteadores são fundamentais para efetivação da integralidade das ações: a promoção da saúde e da integração social em todos os níveis de atenção e o enfrentamento de fragilidades da pessoa idosa, da família e do sistema de saúde.

Para o primeiro eixo, a atenção integral e integrada à saúde da pessoa idosa deverá ser estruturada embasada nos direitos, habilidades, necessidades e preferências do usuário, nos moldes de uma linha de cuidados que vise 
facilitar o acesso a todos os níveis de atenção. O cuidado prestado deve ser realizado considerando-se os distintos fatores que interagem na saúde dos idosos- aspectos físicos, psicológicos, ambientais e sociais- o que demanda uma abordagem global, interdisciplinar e multidimensional orientadas para a promoção da autonomia e independência da pessoa idosa, estimulando-a ao autocuidado.

No segundo eixo, a Política (BRASIL, 2006) propõe a avaliação funcional individual e coletiva como instrumento gerencial para o estabelecimento das ações de atenção. A partir da avaliação funcional coletiva é possível determinar-se a pirâmide de risco funcional da população idosa assistida pelas Unidades Básicas de Saúde, identificando-se a proporção de idosos: independentes, aqueles que já possuem alguma incapacidade funcional para atividades básicas da vida diária, aqueles que apresentam alta dependência funcional, os acamados e os que vivem em Instituições de Longa Permanência para Idosos. Os idosos que mesmo independentes, apresentem alguma dificuldade nas atividades instrumentais de vida diária,deverão ser acompanhados com maior freqüência pelos profissionais de saúde, por serem considerados idosos potenciais para o desenvolvimento de fragilidade (BRASIL, 2006).

Considera-se idoso frágil ou em situação de fragilidade aquele que: esteve hospitalizado recentemente por qualquer razão, encontra-se acamado, vivencie situações de violência doméstica, apresente doenças causadoras de incapacidade funcional, encontra-se com pelo menos uma incapacidade funcional básica, viva em Instituições de Longa Permanência para Idosos ou que possua 75 anos ou mais de idade (BRASIL, 2006).

A partir do reconhecimento das condições de fragilidade pela avaliação funcional individual e coletiva, serão avaliados os recursos locais que permitam seu manejo, com vistas a fomentar uma rede de solidariedade para com o idoso frágil e sua família e promover a reinserção do idoso frágil em sua comunidade. Ainda, por meio da avaliação funcional, é possível identificar a rede de suporte social do idoso e suas necessidades objetivando facilitar o cuidado domiciliar, estabelecer parcerias com os cuidadores informais e prevenir o cansaço e desgaste das pessoas que cuidam (BRASIL, 2006).

\section{CONCLUSÃO}

O crescimento demográfico mundial da população idosa exige a preparação adequada dos países para atendimento às múltiplas demandas. Reconhecendo-se a importante influência das experiências de vida e interação entre diversos fatores na determinação da heterogeneidade nos processos de envelhecer dos indivíduos, é fundamental a garantia aos idosos de seus direitos, e consideração à suas necessidades, preferências e habilidades, visando implementação de estratégias e equiparação de oportunidades de acesso à saúde, segurança e participação ativa em seu meio social.

Atenção especial deve ser dada aos idosos frágeis, na manutenção e melhoria de sua capacidade funcional e no apoio à sua rede informal de cuidados. Deve-se reconhecer a importância da família no desenvolvimento social, porém ressaltando-se que os serviços prestados pelas famílias e pelas comunidades não devem substituir um sistema eficaz e acessível de saúde pública (ONU, 2003). Para tanto, iniciativas como a assistência domiciliar e outras alternativas à institucionalização devem ser fortalecidas, bem como a capacitação em conteúdos de Gerontologia aos cuidadores formais e profissionais.

Enfim, o aumento da expectativa média de vida precisa ser acompanhado de qualidade de vida, o que demanda uma abordagem ampla, transdisciplinar e intersetorial na atenção aos idosos.

BATISTA, M. P. P.; ALMEIDA, M. H.M.; LANCMAN, S. Public policies for the elderly population: a review with emphasis on healthcare actions. Rev. Ter. Ocup. Univ. São Paulo, v. 22, n. 3, p. 200-207, set./dez. 2011.

\begin{abstract}
Population aging is a worldwide phenomenon. This process poses challenges to be addressed, particularly in developing countries, which requires the execution of Public Policies for this population segment. This article, which integrates the Master's Thesis project entitled "Reflections on elderly companion's daily work at the Programa Acompanhante de Idosos- PAI", presents the main international and national documents aimed to guide the implementation of programs and policies for the elderly, with emphasis on the responsibility actions of the Health Sector. These documents recognize elderly as person of right and encourages their independence, autonomy, social participation, family and community life.
\end{abstract}

KEY WORDS: Health public policy; Policy; Aging, Aged. 
BATISTA, M. P. P., et al. Politicas publicas para a população. Rev. Ter. Ocup. Univ. São Paulo, v. 22, n. 3, p. $200-207$, set./dez. 2011.

\section{REFERÊNCIAS}

BRASIL. Constituição (1988). Constituição da República Federativa do Brasil. Brasília, DF: Senado Federal, 1988.

BRASIL. Decreto $n^{\circ} 1.948$. Regulamenta a Lei $n^{\circ} 8.842$, de 4 de janeiro de 1994, que dispõe sobre a Política Nacional do Idoso, e dá outras providências Brasília, DF, 1996.

BRASIL. Lei $n^{\circ} 10.741$. Dispõe sobre o Estatuto do Idoso e dá outras providências. Brasília, DF, 2003.

BRASIL. Lei n ${ }^{\circ}$ 8.842. Dispõe sobre a Política nacional do idoso cria o Conselho Nacional do Idoso e dá outras providências. Brasília, DF, 1994.

BRASIL. Ministério da Saúde. Portaria 1395/GM. Política de Saúde do Idoso. Brasília, DF, 1999.

BRASIL. Ministério da Saúde. Portaria n ${ }^{\circ} 2.528$. Aprova a Política Nacional de Saúde da Pessoa Idosa. Brasília, DF, 2006

IBGE- Instituto Brasileiro de Geografia e Estatística. Pesquisa Nacional Por Amostra de Domicílios - PNAD 2009. Disponível em: <http://www.ibge.gov.br/home/estatistica/populacao/ trabalhoerendimento/pnad2009/sintese_defaultpdf_domicilios. shtm>.

Recebido para publicação: 13/09/11

Aceito para publicação: 18/11/11
IBGE. - Instituto Brasileiro de Geografia e Estatística . Censo Demográfico 2010. Disponível em: <http://www.censo2010. ibge.gov.br/>

OMS - Organização Mundial da Saúde. Declaração elaborada pelo Grupo de Trabalho da Qualidade de Vida da Organização Mundial da Saúde. Genebra, 1994.

OMS - Organização Mundial da Saúde. 26a Conferência Sanitária Pan-Americana. A Saúde e o Envelhecimento. Washington: Organização Pan-Americana da Saúde, 2002.

OMS - Organização Mundial da Saúde. Envelhecimento Ativo: uma Política de saúde. Brasília: Organização Pan-Americana da Saúde, 2005.

ONU - Organização das Nações Unidas. Plano de ação internacional sobre o envelhecimento. Secretaria Especial dos Direitos Humanos. Brasília, 2003.

SABE- Saúde, bem-estar e envelhecimento. In: LEBRÃO, M. L.L.; DUARTE, Y. A.O. (Org.). O Projeto SABE no Município de São Paulo: uma abordagem inicial. Brasília: Organização PanAmericana da Saúde, 2003. 